\title{
Mechanical Properties Analysis of Polypropylene Biocomposites Reinforced with Curaua Fiber
}

\author{
Sistanley Jones Lima Bispo ${ }^{a}$, Raimundo Carlos Silverio Freire Júnior ${ }^{a *}$, Eve Maria Freire de Aquino ${ }^{a}$ \\ ${ }^{a}$ Programa de Graduação de Engenharia Mecânica, Centro de Tecnologia, \\ Departamento de Engenharia Mecânica, Universidade Federal do Rio Grande do Norte-UFRN, \\ Lagoa Nova, Natal, RN, Brazil
}

Received: June 16, 2015; Revised: July 3, 2015

\begin{abstract}
Over the last few years, great interest has been shown by researchers in presenting alternative proposals for the design and fabrication of materials with good mechanical properties and low cost for a variety of applications. With this in mind, a study was carried out on the addition of curaua fibers in a polypropylene (PP) matrix. These were extruded in the pellets form with curaua fiber content of 5, 10 and $20 \%$ (mass percentage). An injector was used to make the test specimens from these pellets, which were then subjected to mechanical tests (tensile and three point bending), physical and thermal tests (fluidity index and HDT). As a consequence, it was noted that the incorporation of fibers in composites of PP with curaua resulted in an increase in the elastic modulus and tensile strength improving therefore the mechanical properties of these materials. Another important point was to check if there was an increase in heat deflection temperature as the fibers were added. As a result, it has been found that it is feasible to use these materials in industry, facilitating its recycling and improving its final mechanical properties.
\end{abstract}

Keywords: biocomposites, mechanical properties, curaua fibers, polypropylene

\section{Introduction}

As applications and services become more sophisticated, it is frequently necessary to find new materials which can meet all the expectations of the industry, especially when considering the cost, mechanical properties and recyclability of the material.

With this in mind, several researchers ${ }^{1-7}$ made an effort to meet this demand. Taking this into account, the biocomposites materials render themselves as an alternative because of the possibility that they will be recyclable, thereby reducing environmental impact and cost of production.

When considering the biocomposites manufactured from natural fibers and thermoplastic matrix, it has already been established in the literature that with a suitable surface treatment of the fiber or using a graft copolymerization matrix ${ }^{1-3,8}$ and by using an increasing percentage volumetric fiber $^{5,9}$ it is possible to use these materials in small and medium structures even if they are in the form of short fibers ${ }^{4,6-7}$.

One of the natural fibers that is currently showing very promising results ${ }^{10-13}$ is the curaua fiber, showing superior resistance in comparison to other more frequently used natural fibers such as sisal, jute, abaca, kenaf ${ }^{5-9,14}$ and therefore its application in composite manufacturing shows much promise for the future.

It is important to mention that in none of the studies cited above was it found how the increase in curaua fiber content influences the properties of the biocomposites nor was it proven that there was an advantage and at what values there wouldn't be any structural advantage in using natural fibers

*e-mail: freirej@ufrnet.br
Therefore, this paper proposes to manufacture biocomposites based on polypropylene (PP) and reinforced curaua fiber of percentages of 5, 10 and $20 \%$, that are thermally and mechanically tested by performing a comparison between the results and the properties of the base material.

\section{Material and Methods}

Polypropylene homopolymer (KM-6100) was used as a raw material for the development of biocomposites which was supplied by Quattor Petroquímica S/A company at a density of $0.9 \mathrm{~g} / \mathrm{cm}^{3}$ and curaua fibers which were provided (in natura) by the Pematec company, located in the city of Santarém, state of Pará / Brazil.

The biocomposite was developed using a process extrusion, pelletization and injection. The mechanical and thermo-mechanical performance of the biocomposite was determined using the uniaxial tensile test, three point bending and heat deflection temperature. The influence in viscosity of the biocomposite, due to the addition of natural fibers, was evaluated in melt flow index test.

\subsection{Manufacturing process}

The specimens were prepared by mixing polypropylene (PP) pellets with caraua fibers, where composites were obtained in the form of chopped fiber grains of mass percentages of 5,10 and $20 \%$. These values were chosen because of the processing capacity of the extruder. The used extruder is a twin screw co-rotating manufactured by IMACOM, model DR 30:40 with screw diameter of $30 \mathrm{~mm}$ and $\mathrm{L} / \mathrm{D}=40$. 
The extruder speed used was $210 \mathrm{rpm}$ at a temperature average $180{ }^{\circ} \mathrm{C}$.

Before the manufacturing of the curaua fibers biocomposites, they were washed with water to remove impurities, combed, chopped and dried in a kiln. Soon after, they were conditioned in aluminum trays.

The PP (polypropylene) biocomposites, were added during the manufacturing process into the screw conveyor and the chopped fibers through a lateral opening, between the screw and the extruder. Both continued moving towards the entrance of the extruder, where the melting and mixing of elements took place. On leaving the extruder in the shape of a "noodle", through two small holes they then made their way to a cooling tank where they were allowed to solidify. After this process, the noodles were sent to the pelletizing machine. The pellets were homogenized and conditioned in a kiln for a period of 24 hours prior to the manufacture of the test samples.

The polypropylene analyzed in the preparation of the test specimens was processed by the same method used in biocomposites. This procedure was implemented in order guarantee that materials possessing identical characteristics and processing could be compared.

After removing the humidity in a kiln, the grains were deposited in a funnel, then injected into a mold at a pressure of $500 \mathrm{bar}$, at an injection rate of $120 \mathrm{~cm}^{2} / \mathrm{s}$ and working temperature of $180^{\circ} \mathrm{C}$. A "ROMI" type injector was used and the complete cycle between injection and cooling was $30 \mathrm{~s}$. The norm used in the process of molding and extrusion was ISO $527-2^{[15]}$.

\subsection{Analysis of the fluidity index (FI)}

In order to check the degree of thermal degradation caused by the extrusion process it was necessary to analyze the melt flow of the material. This is important because it evaluates the performance of the polymer during processing and reprocessing the extruder, indirectly analyzing the decrease of the molar mass of the specimen to its degradation. The decrease in molar mass occurs with an increased fluidity index and may hinder the recycling of the material.

With this in mind, in addition to the analysis of the fluidity index of the aforementioned material, an evaluation of the "virgin" polypropylene was also carried out at this stage, which serves as a point of comparison for the analysis of degradation and recyclability of biocomposites.

The ASTM D $1238^{[16]}$ norm was used for the analysis of the fluidity index using equipment manufactured by Digitrol, which has an accuracy of $\pm 0.001 \mathrm{~g} / \mathrm{min}$ with a test temperature of $230^{\circ} \mathrm{C}$.

\subsection{Tensile and bending in three points testing}

The tensile and three point bending test were carried out on a universal testing machine manufactured by SHIMADZU with a maximum capacity of $30 \mathrm{~T}$; it was used at a speed of $5 \mathrm{~mm} / \mathrm{min}$ in the tensile test and $1 \mathrm{~mm} / \mathrm{min}$ in the bending test. The norms for the procedure and dimensions of the test specimens were ISO $527-5^{[17]}$ and ISO $178^{[18]}$, for testing the tensile and bending respectively. At least eight test specimens were used for each evaluated case.

\subsection{Analysis of Heat Deflection Temperature (HDT)}

In addition to tensile and three point bending, tests were done to measure the Heat Deflection Temperature (HDT) of the materials, using ISO $75-1^{[19]}$. In this test the specimens are immersed in oil, and loaded with bending stress of $450 \mathrm{kPa}$, which applies a heating rate of $2^{\circ} \mathrm{C} / \mathrm{min}$. Hereupon, it evaluates the temperature at which the specimen reaches a deflection of $0.34 \mathrm{~mm}$ which is called heat deflection temperature. The equipment used for this test was HDT 300 VICAT - CEAST.

\section{Results and Discussion}

\subsection{Analysis of fluidity index (FI)}

As previously mentioned, an increase in fluidity index may hinder the recyclability of polypropylene or biocomposites, due to their decreased molar mass and consequent degradability. Thus, for the biocomposites analyzed in the present study, the use of natural fiber did not alter the fluidity index compared with virgin polypropylene (Table 1). This table also shows that polypropylene degrades after the extrusion process, given that its fluidity index increased from 0.32 to $0.51 \mathrm{~g} / \mathrm{min}$ (increase of 59\%). Moreover, the use of $20 \%$ curaua fiber lowered the index to 0.24 .

This result demonstrates that despite the reduced molar mass (indirectly measured by this test) during the extrusion process, the addition of natural fiber compensates the decline in viscosity, thereby allowing its reuse.

\subsection{Tensile strength test}

The tensile strength test results of these biocomposites showed that test specimens of all the biocomposite specimens ruptured and only those of the polypropylene specimens did not. Furthermore, a slight difference occurred between the test specimen results of each case analyzed, except for the value of rupture deformation, which exhibited wide-ranging values.

The results between the biocomposites and polypropylene showed an increase in mechanical properties (strength and stiffness) and a rise in fiber percentage. This finding is presented in Table 2 and Figure 1, which show the mean behavior of these materials for each situation analyzed. According to these values, the most significant increment occurred for the $20 \% \mathrm{PP} /$ curaua biocomposite compared to polypropylene, which obtained a $151 \%$ increase in modulus of elasticity (from 1.08 GPa to $2.72 \mathrm{GPa}$ ). In relation to strength, the greatest increase also occurred for the $20 \% \mathrm{PP} /$ curaua biocomposite, with a $20 \%$ rise in maximum tensile strength (from 25.9 $\mathrm{MPa}$ to $31.2 \mathrm{MPa}$ ).

Table 1. Average values of the pellets submitted to the FI analysis.

\begin{tabular}{cc}
\hline Material & Fluidity Index (g/min) \\
\hline Polypropylene "virgin" & 0.320 \\
Polypropylene & 0.510 \\
PP/Curaua 5\% & 0.371 \\
PP/Curaua 10\% & 0.370 \\
PP/Curaua 20\% & 0.240 \\
\hline
\end{tabular}


Another important point related to Table 2 is the fact that the increase in strength (maximum stress) did not occur linearly, since maximum stress values were practically the same for biocomposites with 5 and $10 \%$ curaua fiber. This result may be related to the formation of stress concentration points on the fiber-matrix interface, thereby masking the greater strength and stiffness caused when an increase in fiber content is not sufficiently high.

The formation of stress concentration points was confirmed by microstructural SEM analysis in the final fracture region of the material (Figure 2), where areas with greater biocomposite deformation (dark area) are observed around the fibers.

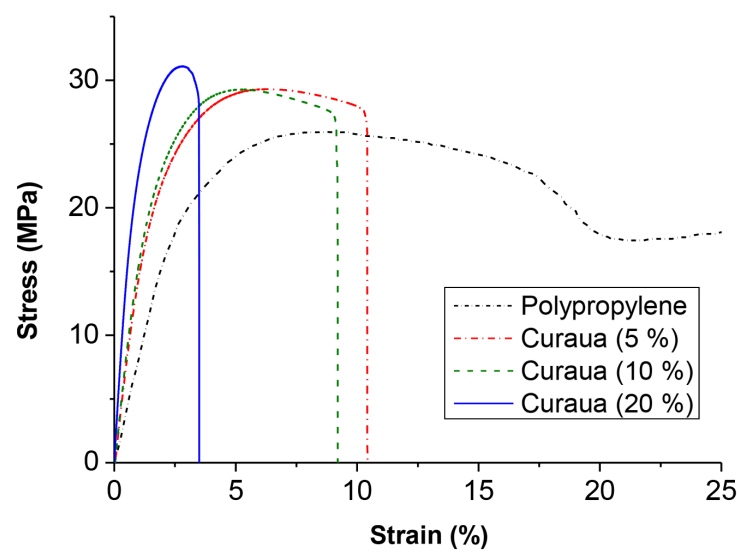

Figure 1. Average stress strain curves of polypropylene and biocomposites.

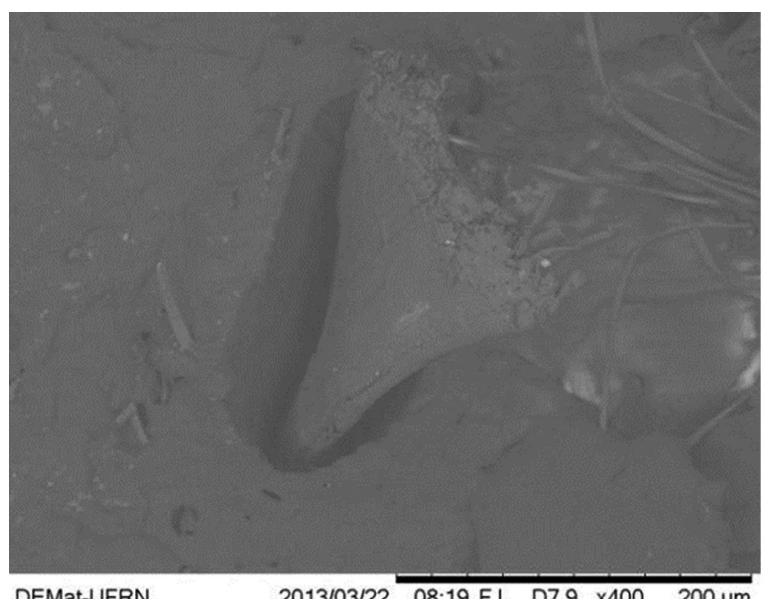

DEMat-UFRN 2013/03/22

(a)

\subsection{Three-point bend test}

In contrast to tensile tests, none of the specimens ruptured during flexural testing, but all tests exhibited a maximum stress value. There was also little dispersion between specimens. An example can be seen in Figure 3 for the polypropylene biocomposite with $20 \%$ curaua fiber.

A comparative study between results showed an increase in strength and stiffness (Table 3 and Figure 4) with the addition of fiber to the biocomposite, as occurred in tensile testing. The difference was highest for stiffness (modulus of elasticity), with an increase of $105 \%(1.09 \mathrm{GPa}$ in PP to $2.24 \mathrm{GPa}$ in the biocomposite with $20 \%$ curaua fiber). A similar result was obtained by Raman et al. ${ }^{20}$ for a PP composites reinforced with jute fibers.

With respect to strength, the increase was $23.2 \%$, where maximum stress rose from $40.8 \mathrm{MPain}$ polypropylene to $50.3 \mathrm{MPa}$ in the biocomposite with $20 \%$ curaua fiber.

It was also determined that the increase in strength (maximum tensile) and stiffness (modulus of elasticity) did not occur linearly, since fiber percentages of 5 and $10 \%$ exhibited similar mechanical properties. Once again, the effect of stress concentration caused by adding fibers to polypropylene may have masked the increase in the mechanical properties of the biocomposite.

\subsection{Heat deflection temperature (HDT) test}

Analysis of heat deflection temperature is important for thermoplastic applications (or thermoplastic-based materials) in industry, since its values determine, among others, the capacity of this material to withstand a sterilization temperature (around $121^{\circ} \mathrm{C}$ ), or whether it can be used in hot-filling processes of pasteurized products with working temperatures between $75^{\circ} \mathrm{C}$ and $100^{\circ} \mathrm{C}$.

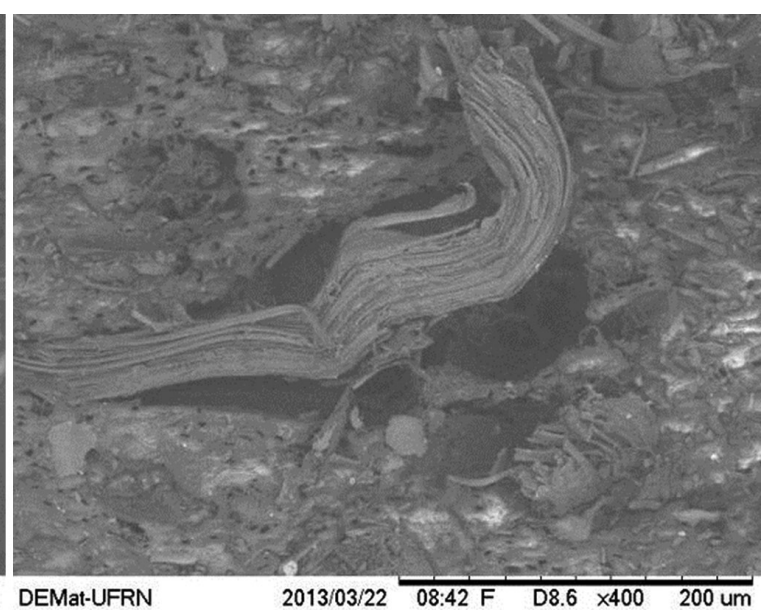

(b)

Figure 2. Scanning electron microscopy of (a) $5 \% \mathrm{PP} /$ Curaua and (b) $20 \% \mathrm{PP} /$ Curaua.

Table 2. Mechanical properties of polypropylene and the biocomposite obtained in the tensile test.

\begin{tabular}{cccc}
\hline Material & Maximum Stress (MPa)* & Rupture Strain (\%)* & Elastic Modulus (GPa)* $^{*}$ \\
\hline Polypropylene & $25.94(0.3)$ & - & $1.08(0.04)$ \\
PP/Curaua 5\% & $29.47(0.5)$ & $10.34(2.2)$ & $1.42(0.05)$ \\
PP/Curaua 10\% & $29.35(0.8)$ & $9.11(0.9)$ & $1.54(0.06)$ \\
PP/Curaua 20\% & $31.21(0.2)$ & $3.48(0.3)$ & $2.72(0.03)$ \\
\hline
\end{tabular}

*The values in parentheses correspond to the standard deviation. 
Table 3. Mechanical properties of polypropylene and the biocomposite obtained in the bending test.

\begin{tabular}{ccc}
\hline Material & Maximum Flexural Stress (MPa)* & Flexural Modulus (GPa)* \\
\hline Polypropylene & $40.84(0.5)$ & $1.09(0.04)$ \\
PP/curaua 5\% & $43.38(0.7)$ & $1.23(0.05)$ \\
PP/curaua 10\% & $43.51(1.5)$ & $1.30(0.06)$ \\
PP/curaua 20\% & $50.27(0.3)$ & $2.24(0.03)$ \\
\hline
\end{tabular}

*The values in parentheses correspond to the standard deviation.

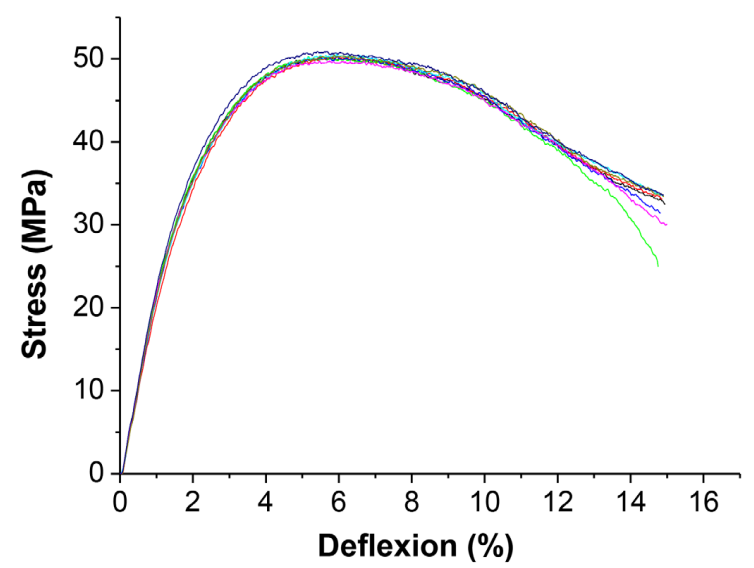

Figure 3. Stress deflexion curves of biocomposite with $20 \%$ of curaua fiber.

Figure 5 shows heat deflection temperature (HDT) values obtained for the biocomposites and polypropylene. Only $20 \%$ curaua fiber obtained a rise in HDT, increasing from $75.30176 \mathrm{C}$ (polypropylene) to $97^{\circ} \mathrm{C}$ (increase of $28.8 \%$ ).

This result is important, since it demonstrates that the use of curaua fiber improves the thermal stability of the composite, meaning it can be used in applications with higher temperatures. Jarukumjorn and Suppakarn ${ }^{21}$ found a similar effect to that observed for the curaua fibers studied here, demonstrating that the addition of sisal fibers along with polypropylene P700J increases heat deflection temperature.

With respect to a possible industrial application, the results obtained for the biocomposite with $20 \%$ curaua fibers are also promising, since it can be used, for example, in the manufacture of containers for hot-filling pasteurization. However, if a sterilized product were to be used, a study with higher fiber percentages in the biocomposite would be needed.

\section{Conclusions}

Based on the results obtained in the present study, the following conclusions can be drawn:

- The addition of curaua fibers in PP reduces the effect caused by thermal degradation of polypropylene during the extrusion process;

\section{References}

1. Herrera-Franco PJ and Valadez-Gonzalez A. A study of the mechanical properties of short natural-fiber reinforced composites. Composites. Part B, Engineering. 2005; 36(8):597-608. http:// dx.doi.org/10.1016/j.compositesb.2005.04.001.

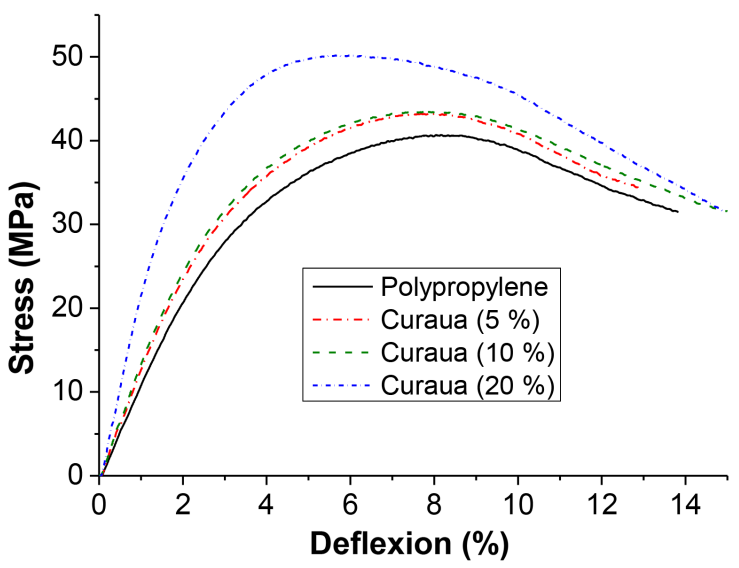

Figure 4. Average stress deflexion curves of polypropylene and biocomposites.

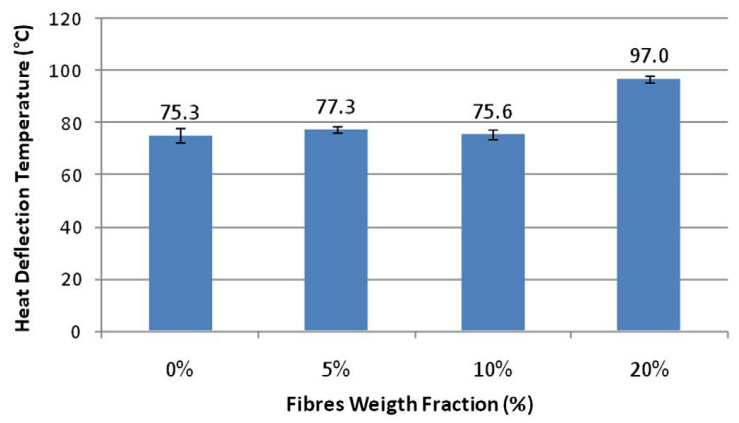

Figure 5. Heat deflection temperature of the curaua composites.

- In the three-point bend test the addition of curaua fiber had a greater effect on stiffness than on strength in the biocomposites;

- Only a 20\% increase in curaua fibers had a significant effect on the mechanical properties analyzed, this was verified for both the tensile tests and for the three-point bending tests;

- In the HDT test the addition of curaua fibers increased the heat deflection temperature of the composite, improving its thermal stability and providing a quality material in high-temperature applications.

2. Lee BH, Kim HS, Lee S, Kim HJ and Dorgan JR. Biocomposites of kenaf fibers in polyactide: Role of improved interfacial adhesion in the carding process. Composites Science and Technology. 2009; 69(15-16):2573-2579. http://dx.doi. org/10.1016/j.compscitech.2009.07.015. 
3. Barreto ACH, Rosa DS, Fechine PBA and Mazzetto SE. Propeties of sisal fibers treated by alkali solution and their application into cardanol-based biocomposites. Composites. Part A, Applied Science and Manufacturing. 2011; 42(5):492-500. http://dx.doi. org/10.1016/j.compositesa.2011.01.008.

4. Abdelmouleh M, Boufi S, Belgacem MN and Dufresne A. Short natural-fibre reinforced polyethylene and natural rubber composites: effect of silane coupling agents and fibres loading. Composites Science and Technology. 2007; 67(7-8):1627-1639. http://dx.doi.org/10.1016/j.compscitech.2006.07.003.

5. Rao KMM, Rao KM and Prasad AVR. Fabrication and testing of natural fibre composites: vakka, sisal, bamboo and banana. Materials \& Design. 2010; 31(1):508-513. http://dx.doi. org/10.1016/j.matdes.2009.06.023.

6. Castro DO, Ruvolo-Filho A and Frollini E. Materials prepared from biopolyethylene and curaua fibers: Composites from biomass. Polymer Testing. 2012; 31(7):880-888. http://dx.doi. org/10.1016/j.polymertesting.2012.05.011.

7. Gutiérrez MC, De Paoli M-A and Felisberti MI. Biocomposites based on cellulose acetate and short curauá fibers: effect of plasticizers and chemical treatments of the fibers. Composites. Part A, Applied Science and Manufacturing. 2012; 43(8):13381346. http://dx.doi.org/10.1016/j.compositesa.2012.03.006.

8. Malkapuram R, Kumar V and Negi YS, Negi YS. Recent development in natural fiber reinforced polypropylene composites. Journal of Reinforced Plastics and Composites. 2009; 28(10):1169-1189. http://dx.doi.org/10.1177/0731684407087759.

9. $\mathrm{Ku} \mathrm{H}$, Wang H, Pattarachaiyakoop $\mathrm{N}$ and Trada $\mathrm{M}$. A review on the tensile properties of natural fiber reinforced polymer composites. Composites. Part B, Engineering. 2011; 42(4):856873. http://dx.doi.org/10.1016/j.compositesb.2011.01.010.

10. Silva RV and Aquino EMF. Curaua fiber: a new alternative to polymeric composites. Journal of Reinforced Plastics and Composites. 2008; 27(1):103-112. http://dx.doi. org/10.1177/0731684407079496.

11. Rodrigues LPS, Silva RV and Aquino EMF. Effect of accelerated environmental aging on mechanical behavior of curaua/glass hybrid composite. Journal of Composite Materials. 2012; 46(17):2055-2064. http://dx.doi.org/10.1177/0021998311429880.
12. Spinacé MAS, Fermoseli KKG and De Paoli M-A. Recycled polypropylene reinforced with curaua fibers by extrusion. Journal of Applied Polymer Science. 2009; 112(6):3686-3694. http://dx.doi.org/10.1002/app.29683.

13. Egute NS, Forster PL, Parra DF, Fermino DM, Santana S and Lugão AB. Mechanical and thermal properties of polypropylene composites with curauá fibre irradiated with gamma radiation. In: International Nuclear Atlantic Conference; 2009; Rio de Janeiro, Brazil. Rio de Janeiro: Associação Brasileira de Energia Nuclear; 2009. 8 p.

14. Espert A, Vilaplana F and Karlsson S. Comparison of water absorption in natural cellulosic fibres from wood and one-year crops in polypropylene composites and its influence on their mechanical properties. Composites. Part A, Applied Science and Manufacturing. 2004; 35(11):1267-1276. http://dx.doi. org/10.1016/j.compositesa.2004.04.004.

15. International Organization for Standardization - ISO. ISO 5272: Determination of tensile properties - part 2: test conditions for moulding and extrusion plastics. Geneva: ISO; 2012.

16. American Society for Testing and Materials-ASTM. ASTMD 1238: Standard test method for melt flow rates of thermoplastics by extrusion plastometer. West Conshohocken: ASTM; 2010.

17. International Organization for Standardization - ISO. ISO 5275: Determination of tensile properties - Part 5: test conditions for unidirectional fibre-reinforced plastic composites. Geneva: ISO; 2009.

18. International Organization for Standardization - ISO. ISO 178: Determination of flexural properties. Geneva: ISO; 2010.

19. International Organization for Standardization - ISO. ISO 75-1: Determination of temperature of deflection under load - part 1: general test method. Geneva: ISO; 2004.

20. Raman R, Hasan M, Huque M and Islam N. Physico-mechanical properties of jute fiber reinforced polypropylene composites. Journal of Reinforced Plastics and Composites. 2010; 29(3):445455. http://dx.doi.org/10.1177/0731684408098008.

21. Jarukumjorn K and Suppakarn N. Effect of glass fiber hybridization on properties of sisal fiber - polypropylene composites. Composites. Part B, Engineering. 2009; 40(7):623-627. http:// dx.doi.org/10.1016/j.compositesb.2009.04.007. 